\title{
High Power Ion Heating in Helium and Hydrogen Plasmas for Advanced Plasma Thrusters
}

\author{
By Akira ANDO, Tatsuya HAGIWARA, Masakazu DOMON, and Takahiro TAGUCHI \\ Department of Electrical Engineering, Tohoku University, Sendai, Japan
}

(Received May 2nd, 2008)

\begin{abstract}
High power ion cyclotron resonance heating is performed in a fast-flowing plasma operated with hydrogen and helium gases. Ion heating is clearly observed in hydrogen plasma as well as in helium plasma. The resonance region of magnetic field is broader and wave absorption efficiency is higher in hydrogen plasma than those in helium plasma. The thermal energy of the heated ions is converted to the kinetic energy of the exhaust plume by passing through a diverging magnetic nozzle set in a downstream region. In the magnetic nozzle energy conversion occurred as keeping the magnetic moment constant, but some discrepancy was observed in larger gradient of magnetic field. The kinetic energy of the exhaust plume is successfully controlled by an input power of radio-frequency wave, which is one of the key technologies for the Variable Specific Impulse Magnetoplasma Rocket (VASIMR) type plasma thruster.
\end{abstract}

Key Words: Ion cyclotron heating, Magnetoplasma rocket, VASIMR, Magnetic nozzle

\section{Nomenclature}

\begin{tabular}{|c|c|}
\hline$I_{d}$ & : discharge current \\
\hline$B$ & : magnetic field strength \\
\hline$B_{\mathrm{U}}$ & : magnetic field in upstream region \\
\hline$B_{\mathrm{D}}$ & : magnetic field at cyclotron resonance \\
\hline$B_{\mathrm{N}}$ & $\begin{array}{l}\text { magnetic field in the magnetic nozzle } \\
\text { exit }\end{array}$ \\
\hline$\omega$ & : angular frequency of excited RF wave \\
\hline$\omega_{c i}$ & $\begin{array}{l}\text { angular frequency of ion cyclotron } \\
\text { motion }\end{array}$ \\
\hline$W_{\perp}$ & $\begin{array}{l}\text { kinetic energy corresponding to the } \\
\text { perpendicular motion to magnetic field }\end{array}$ \\
\hline$W_{/ /}$ & $\begin{array}{l}\text { kinetic energy corresponding to the } \\
\text { parallel motion to magnetic field }\end{array}$ \\
\hline$T_{i \perp}$ & $\begin{array}{l}\text { ion temperature corresponding to the } \\
\text { perpendicular motion to magnetic field }\end{array}$ \\
\hline$T_{i / /}$ & $\begin{array}{l}\text { ion temperature corresponding to the } \\
\text { parallel motion to magnetic field }\end{array}$ \\
\hline$n_{e}$ & : electron density \\
\hline$T_{e}$ & : electron temperature \\
\hline$P_{\mathrm{RF}}$ & : input RF power \\
\hline$Z$ & : axial position \\
\hline$\mu$ & : magnetic moment \\
\hline
\end{tabular}

\section{Introduction}

Near-future space exploration missions need further investigation of advanced electric propulsion systems, where high density plasma production and acceleration technique are inevitable. One feature of the advanced space propulsion system is the ability to vary its specific impulse so that it can be operated in a mode with suitable propellant utilization and thrust performance. An ion heating and magnetic nozzle acceleration in a fast-flowing plasma attract much attention in such advanced electric propulsion systems. In the VAriable Specific Impulse Magnetoplasma Rocket (VASIMR) project, it is proposed to control a ratio of specific impulse to thrust at constant power. ${ }^{1)}$ 2) This engine utilizes a combined system of an ion cyclotron heating and a magnetic nozzle, where a flowing plasma is heated by ion cyclotron range of frequency (ICRF) wave power and plasma thermal energy is converted to flow energy via a diverging magnetic nozzle. Utilization of a magnetic nozzle in electric propulsion systems will yield practical benefit not only for avoiding direct contact between a plasma and wall at the exit but for controlling a specific impulse by adjusting the nozzle shape.

Though ion heating technique has been precisely investigated both theoretically and experimentally in many magnetically-confined fusion plasma researches, direct ion heating for fast flowing plasmas by radio-frequency (RF) waves were considered to be difficult, because ions pass quickly through the resonance region only once and strong absorption of RF waves is necessary for the one-pass absorption. Moreover, a charge-exchange energy loss by neutral gas deteriorates the heating evidence in a weakly-ionized plasma.

We have demonstrated for the first time the combined experiments of ion cyclotron resonance heating and acceleration in a magnetic nozzle using a fast-flowing plasma in the HITOP device. ${ }^{3)-6)}$ A magneto-plasmadynamic arcjet (MPDA) was used as a fast-flowing plasma source and operated with quasi-steady duration of $1 \mathrm{~ms}$, which eliminated excess inlet of neutral gas flux and reduced charge-exchange loss. When RF waves were excited by a helically-wound antenna, plasma thermal energy $W_{\perp}$ and ion temperature $T_{\mathrm{i} \perp}$ clearly increased during the RF pulse. Here, $W_{\perp}$ is the kinetic energy 
corresponding to the perpendicular motion to the magnetic field, which hereafter will be referred as "perpendicular energy." Also, $T_{\mathrm{i} \perp}$ expresses the ion temperature corresponding to the perpendicular motion. Hereinafter the suffix $\perp$ and $/ /$ are used in the paper to express perpendicular and parallel component to the axial magnetic field, respectively. As ions are accelerated perpendicular direction to magnetic field by the cyclotron heating, conversion from perpendicular to parallel energy component along the field line is inevitable for the thruster application. We also observed the energy conversion in a diverging magnetic nozzle after the ICRF heating. ${ }^{7)}$ The effects of the magnetic nozzle were investigated and demonstrated that parallel energy of exhausting plasma was changed by controlling the input RF power $P_{\mathrm{RF}}$ only. It was confirmed experimentally that the feature of variable $I_{\mathrm{sp}}$ control demanded in the VASIMR-type thruster can be realized not only by shaping the magnetic nozzle configuration but by controlling the RF input power.

In this research we have performed ICRF heating experiments in hydrogen as well as helium as propellant gas in order to obtain higher exhaust velocity of ions and to achieve ICRF heating in higher density region. It is effective to use lightweight ions for higher cyclotron frequency with lower magnetic field in order to operate higher density plasmas. The exhaust velocity is also expected to be larger in lightweight ions. Fundamental characteristics of resonant magnetic field and dependence on RF input power and plasma density were obtained. We have also investigated effects of a diverging magnetic nozzle on a plasma heated by ICRF heating. The ion thermal energy of the exhaust plasma plume decreased and its kinetic energy increased in a diverging magnetic nozzle. The exhaust energy was controlled not only by changing the nozzle shape but by changing the heating power without varying the nozzle shape.

\section{Experimental setup}

Experiments were carried out in the HITOP device of Tohoku University. ${ }^{8)-10)}$ Schematic view of the device is shown in Fig.1. It consists of a large cylindrical vacuum chamber (diameter $D=0.8 \mathrm{~m}$, length $L=3.3 \mathrm{~m}$ ) with eleven main and six auxiliary magnetic coils, which generate a uniform magnetic field up to $0.1 \mathrm{~T}$. Various types of magnetic field configuration can be formed by adjusting an external coil current.

A high power, quasi-steady magneto-plasma-dynamic thruster (MPDT) was installed at one end-port of the HITOP. It has a coaxial structure with a center tungsten rod cathode $(10 \mathrm{~mm}$ in diameter $)$ and an annular molybdenum anode $(30 \mathrm{~mm}$ in diameter). A discharge current $I_{\mathrm{d}}$ up to $10 \mathrm{kA}$ is supplied by a pulse-forming network (PFN) system with the quasi-steady duration of $1 \mathrm{~ms}$. The current $I_{\mathrm{d}}$ is kept nearly constant during a discharge with a typical voltage of $200 \mathrm{~V}-300 \mathrm{~V}$ and can be controlled by varying a charging voltage of capacitor banks of the PFN power-supply. It can generate a high density (more than $10^{20} \mathrm{~m}^{-3}$ ) plasma. As the produced plasma density is rather high in the MPDT, a stainless mesh with floating potential was set at the exit region for lower density experiments (lower than $10^{18} \mathrm{~m}^{-3}$ ), which was useful to eliminate unexpected current flow path in the plasma.

We installed a right-handed helically-wound antenna at $Z=0.6 \mathrm{~m}$ downstream of the MPDT in the chamber. Here, $Z=0$ corresponds to the position of the MPDT cathode tip. $\mathrm{RF}$ waves can be excited in the direction downstream of the antenna preferentially with azimuthal mode numbers of $m=-1$. RF power was supplied with two types of sources. One was an inverter-type RF wave amplifier operated with a pulsed mode. It was operated with a frequency from $0.1 \mathrm{MHz}$ to $0.5 \mathrm{MHz}$ with a pulse length of $0.5 \mathrm{~ms}$ and an input power up to $20 \mathrm{~kW}$ in the experiments. The other was a vacuum tube RF amplifier operated with a 50-ohm matching circuit in the range of frequency from $0.5 \mathrm{MHz}$ to $1.0 \mathrm{MHz}$. The pulse length was limited to $0.1 \mathrm{~ms}$ and an input power up to $10 \mathrm{~kW}$ was supplied.

A diamagnetic coil was set at $Z=2.23 \mathrm{~m}$ to measure plasma thermal energy $W_{\perp}$. Ion temperature and ion energy distribution function were measured by electrostatic energy analyzers (EEA), which were set at the diamagnetic coil position of $Z=2.33 \mathrm{~m}$ and $Z=3.13 \mathrm{~m}$. The EEA consists of a metal plate with a small circular hole and three grids. Ions get through the small hole and are reflected by a retarding voltage applied between the grids. By facing the normal of the hole parallel and perpendicular to the plasma flow, we can obtain both components of ion temperature, $T_{\mathrm{i} / /}$ and $T_{\mathrm{i} \perp}$.

In the experiments the magnetic field configuration was set as a magnetic-beach type with a constant $B_{\mathrm{U}}(=0.1 \mathrm{~T})$ at the antenna position and a variable $B_{\mathrm{D}}$ (corresponding to ion cyclotron resonance condition) at the diamagnetic coil

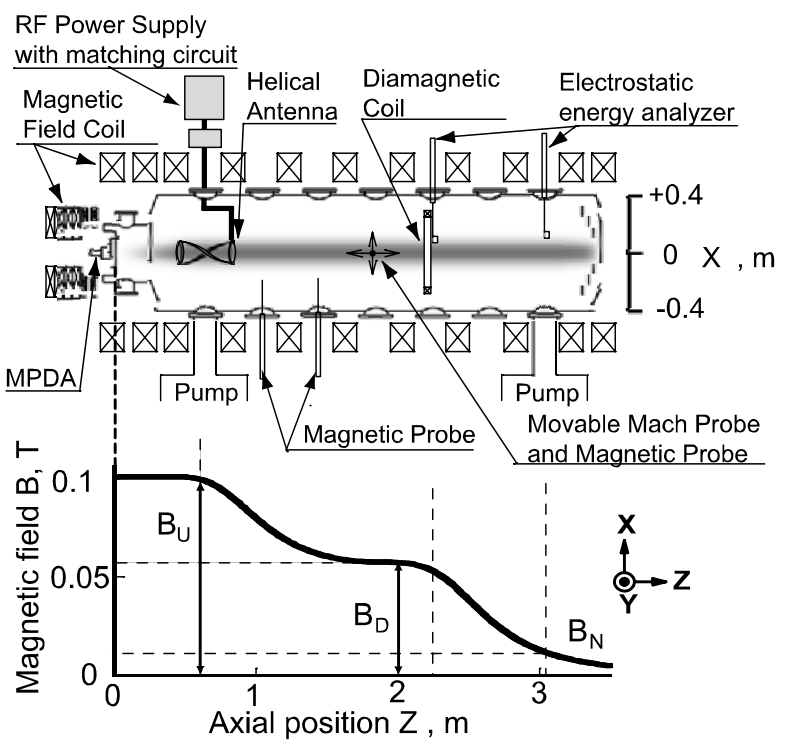

Fig. 1. Schematic of the HITOP device. Magnetic field with magnetic beach configuration is also shown. 
position. The magnetic nozzle shape was also arranged by varying $B_{\mathrm{N}}$ (corresponding to the EEA position of downstream region).

\section{Experimental results}

\subsection{ICRF heating in hydrogen plasmas}

Figure 2 shows typical waveforms of diamagnetic coil signals $W_{\perp}$ obtained in hydrogen plasmas. It drastically increased during the RF excitation, as was also observed in helium plasmas. The maximum value of $W_{\perp}$ increased almost linearly with $P_{\mathrm{RF}}$. Ion temperatures of parallel and perpendicular components are measured by an electrostatic energy analyzer to confirm the ion heating. The ion temperature $T_{\mathrm{i} \perp}$ increased from $2 \mathrm{eV}$ to nearly $60 \mathrm{eV}$ with the electron density of $1.5 \times 10^{17} \mathrm{~m}^{-3}$.

In order to clarify the ion cyclotron resonance heating of hydrogen, we varied the magnetic field $B_{\mathrm{D}}$ in the resonance region. Figure 3 shows the obtained dependences of $\Delta W_{\perp} / W_{\perp}$ on the magnetic field $B_{D}$ for different RF frequencies $f_{\mathrm{RF}}$. $B_{\mathrm{D}}$ corresponding to $\omega / \omega_{c i}=1$ for each $f_{\mathrm{RF}}$ are indicated as arrows in the figure. Here $\Delta W_{\perp}$ is the increase value of $W_{\perp}$ when the RF wave was excited. $\Delta W_{\perp} / W_{\perp}$ became large in the region of $B_{\mathrm{D}}$

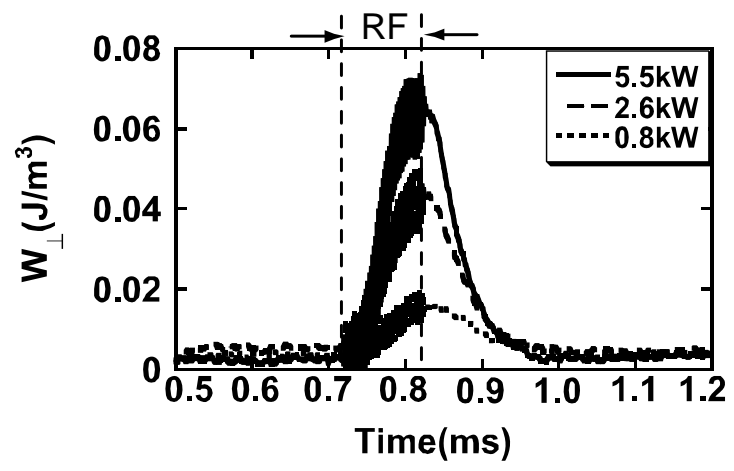

Fig. 2. Time evolutions of $W_{\perp} . P_{\mathrm{RF}}=0.8,2.6$ and $5.5 \mathrm{~kW}$. $f_{\mathrm{RF}}=0.5 \mathrm{MHz} . n_{\mathrm{e}}=1.5 \times 10^{17} \mathrm{~m}^{-3}$. Hydrogen plasma.

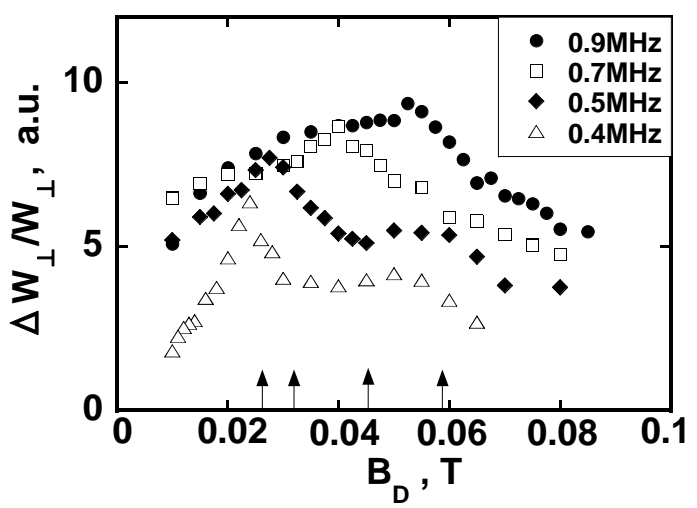

Fig. 3. Dependence of $\Delta W_{\perp} / W_{\perp}$ on the resonance magnetic field $B_{\mathrm{D}}$. Hydrogen plasma. slightly lower than the resonance field of $\omega / \omega_{\mathrm{ci}}=1$. These shifts to lower magnetic field are caused by the Doppler effect due to fast-flowing ions in the plasma. These phenomena are similar to that in helium plasma. The increment of $W_{\perp}$ was, however, observed in broader $B_{\mathrm{D}}$ field than that in helium plasma. One possible reason why the resonance region becomes broad is that the resonance condition was satisfied at different position from the plateau $B_{\mathrm{D}}$ area. Another is the resonance of molecular ions $\mathrm{H}_{2}{ }^{+}$was occurred, where the resonant magnetic field is twice to that of $\mathrm{H}^{+}$ions. Slight increase of the ratio $\Delta W_{\perp} / W_{\perp}$ was observed at $B_{\mathrm{D}}$ of $\omega / \omega_{c i}=1 / 2$ in case of $f_{\mathrm{RF}}=0.4 \mathrm{MHz}$ and $0.5 \mathrm{MHz}$. Further consideration and experiments are necessary for the characteristics of cyclotron resonance condition in hydrogen plasma.

In order to compare the efficiency of ion heating between helium and hydrogen plasmas, we measured the increment ratio $\Delta W_{\perp} / W_{\perp}$ in various densities of plasmas. As the experimental conditions of magnetic field, RF frequency and input power were different in each plasma, it was somewhat difficult to compare the efficiency with each other. We measured magnetic fluctuation signal $\tilde{B}$ using a magnetic probe near the antenna to estimate wave power intensity excited in the plasmas. As the value of $|\tilde{B}|^{2}$ corresponds to power density of the excited waves, the ratio $\Delta W_{\perp} / W_{\perp}$ divided by $|\tilde{B}|^{2}$ is considered to be proportional to the heating efficiency of the RF waves. Figure 4 shows the dependence of $\left(\Delta W_{\perp} / W_{\perp}\right) /|\tilde{B}|^{2}$ on $n_{\mathrm{i}}$ in helium and hydrogen plasmas. As is shown in the figure, the efficiency became smaller with the increase of $n_{\mathrm{i}}$. It is noted that the efficiency was higher in hydrogen plasma than that in helium plasma. ${ }^{11)}$

\subsection{Energy conversion at the magnetic nozzle}

We measured ion temperatures by the EEAs in three types of magnetic nozzle configurations. Figure 5 shows typical EEA signals obtained in helium plasma before and after the nozzle with $B_{\mathrm{D}}(Z=2.33 \mathrm{~m})=57.5 \mathrm{mT}$ and $B_{\mathrm{N}}$ $(Z=3.13 \mathrm{~m})=6.9 \mathrm{mT}$. Strong increase of ion temperature, especially in the perpendicular direction was occurred before the magnetic nozzle. $T_{\mathrm{i} \perp}$ increased from $5 \mathrm{eV}$ to

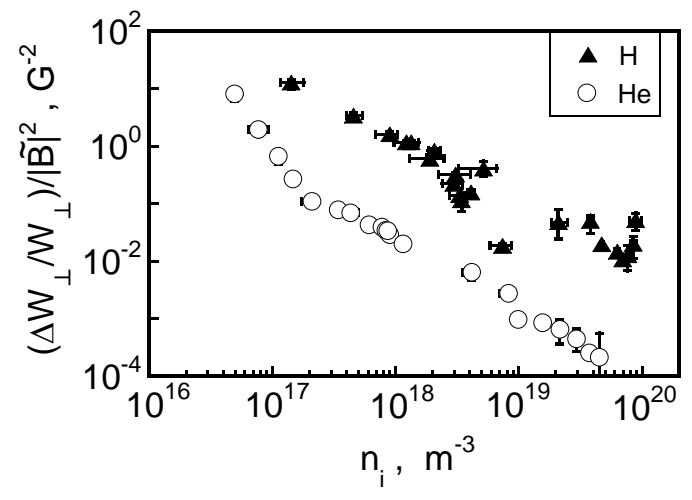

Fig. 4. Dependence of $\Delta W_{\perp} / W_{\perp}$ normalized by $|B|^{2}$ on $n_{\mathrm{i}}$. $f_{\mathrm{RF}}=0.24 \mathrm{MHz}$ in $\mathrm{He}$ plasma and $f_{\mathrm{RF}}=0.41 \mathrm{MHz}$ in $\mathrm{H}$ plasma. 

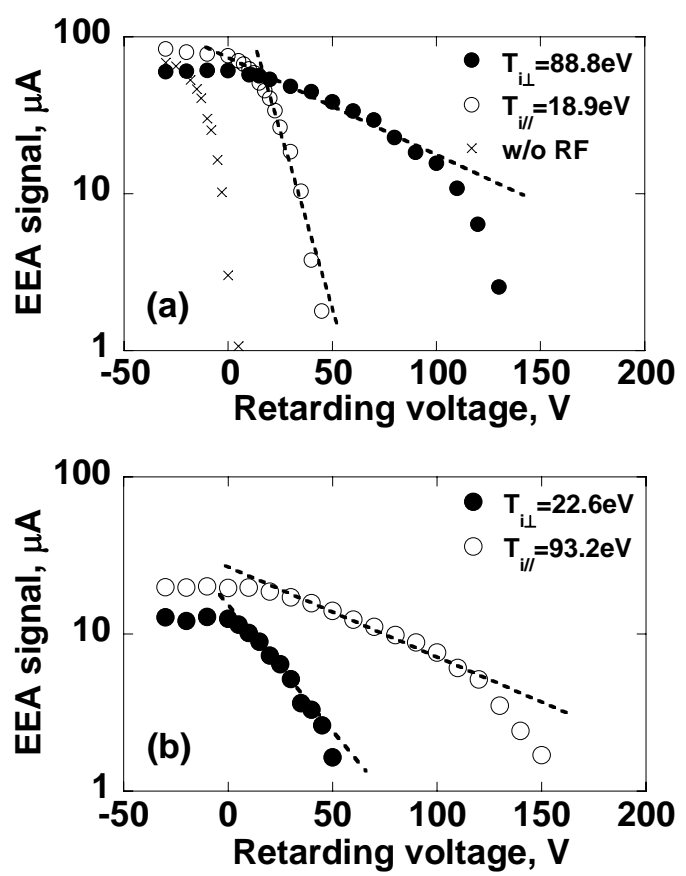

Fig.5. Electrostatic energy analyzer signals measured at (a) $Z=2.33 \mathrm{~m}$ and (b) $Z=3.13 \mathrm{~m}$. He plasma. $P_{\mathrm{RF}}=19 \mathrm{~kW}$, $f_{\mathrm{RF}}=0.24 \mathrm{MHz}, \quad n_{\mathrm{e}}=1.0 \times 10^{17} \mathrm{~m}^{-3}, \quad B_{\mathrm{D}}=57.5 \mathrm{mT}, \quad$ and $B_{\mathrm{N}}=6.9 \mathrm{mT}$

$89 \mathrm{eV}$ with the RF input power of $19 \mathrm{~kW}$. The EEA signal decreased above the retarding voltage of $100 \mathrm{~V}$ as in Fig.5(a). The Larmour radius of helium ions becomes $5 \mathrm{~cm}$ with $T_{\mathrm{i} \perp}=100 \mathrm{eV}$ and $B=57.5 \mathrm{mT}$, which almost equals to the plasma radius. The highly heated ions expanded to outer region and the signal measured at the center position decreased above the retarding voltage of $100 \mathrm{~V}$.

By passing through the diverging magnetic nozzle, increase of $T_{\mathrm{i} / /}$ and decrease of $T_{\mathrm{i} \perp}$ were clearly observed in the analyzer signals. Figure 6 shows an axial profiles of measured $T_{\mathrm{i} \perp}$ and $T_{\mathrm{i} / /}$ in the three magnetic nozzle configurations. It is shown that parallel energy of the exhausting plasma can be controlled by changing the magnetic nozzle configuration.

This energy conversion was occurred due to the conservation law of the magnetic moment, $\mu=W_{\perp} / B=m v^{2} / 2 B$. Profiles of $T_{\mathrm{i} \perp}$ and $T_{\mathrm{i} / /}$ calculated by assuming $\mu=$ const. are also shown in Fig.6 (a) and (b), respectively. It is confirmed that $T_{\mathrm{i} \perp}$ varied so as to keep the magnetic moment constant but some discrepancy was observed in larger gradient of the magnetic field. It was probably caused by a particle motion along magnetic field with larger gradient, because the adiabatic assumption where the magnetic field is kept constant in a single cyclotron motion became invalid.

The parallel energy of exhausting plasma can be changed by controlling the input RF power $P_{\mathrm{RF}}$. Figure 7 shows dependences of $T_{\mathrm{i} \perp}$ and $T_{\mathrm{i} / /}$ on $P_{\mathrm{RF}}$ measured at $Z=2.33 \mathrm{~m}$ (before the magnetic nozzle) and $Z=3.13 \mathrm{~m}$ (after the magnetic nozzle). As shown in Fig.7(a), $T_{\mathrm{i} \perp}$ increased linearly with the increase of $P_{\mathrm{RF}}$, whereas $T_{\mathrm{i} / /}$ slightly

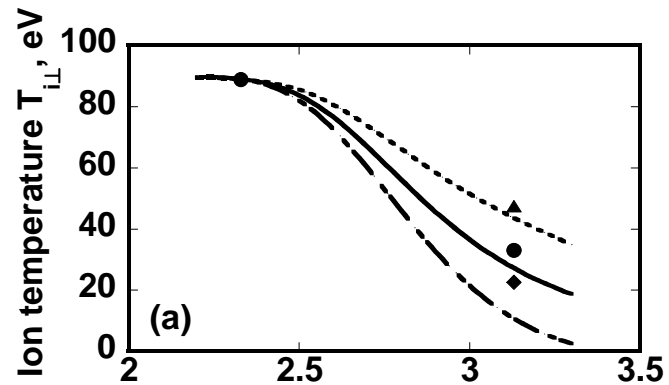

Axial position Z, m

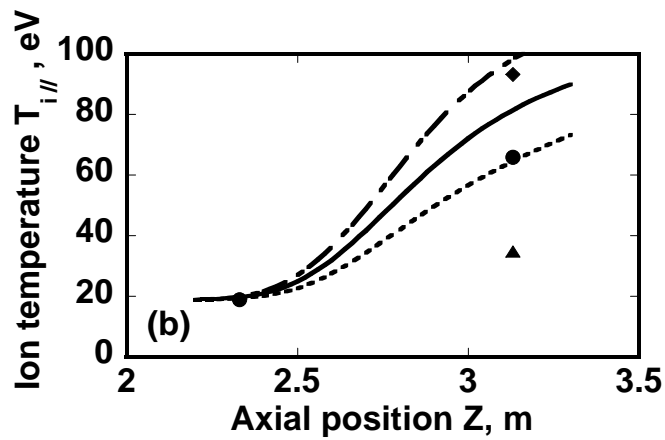

Fig. 6. Axial profile of (a) $T_{\mathrm{i} \perp}$ and (b) $T_{\mathrm{i} / /}$ in the three magnetic nozzle configurations. Lines are calculated ones assuming $\mu=$ const. Closed triangles: $B_{\mathrm{N}}=28.1 \mathrm{mT}$, closed circles: $B_{\mathrm{N}}=17.2 \mathrm{mT}$, closed diamonds: $B_{\mathrm{N}}=6.9 \mathrm{mT}$
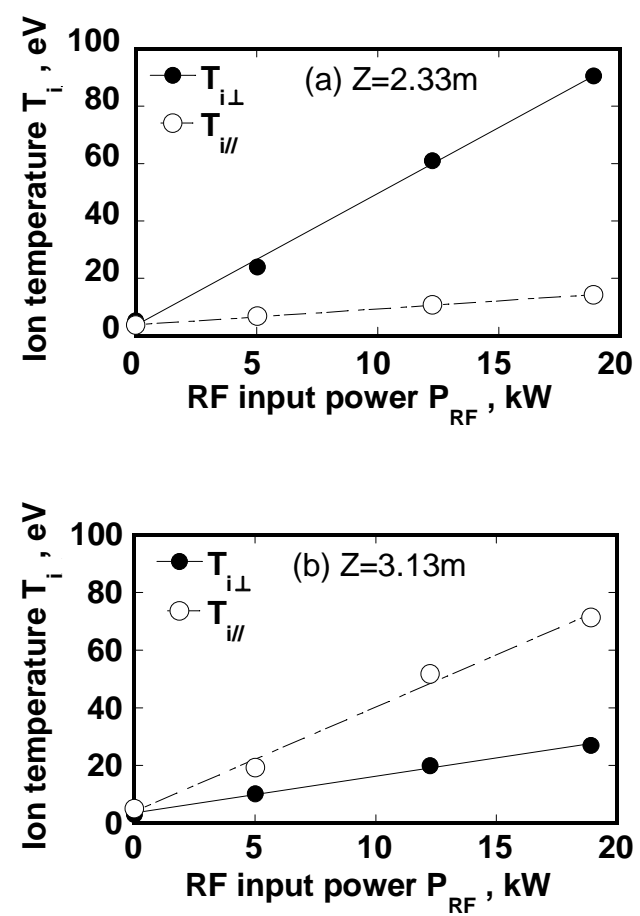

Fig. 7. Dependence of $T_{\mathrm{i} \perp}$ (closed circles) and $T_{\mathrm{i} / /}$ (open circles) on RF input power measured at (a) $Z=2.33 \mathrm{~m}$ and (b) $Z=3.13 \mathrm{~m} . f_{\mathrm{RF}}=0.24 \mathrm{MHz}, n_{\mathrm{e}}=1.0 \times 10^{17} \mathrm{~m}^{-3}, B_{\mathrm{D}}=57.5 \mathrm{mT}$, and $B_{\mathrm{N}}=17.2 \mathrm{mT}$.

increased with $P_{\mathrm{RF}}$ in the ion heating region $(Z=2.33 \mathrm{~m})$. After the energy conversion in the magnetic nozzle, $T_{\mathrm{i} / /}$ strongly increased linearly with the increase of $P_{\mathrm{RF}}$ as 
shown in Fig.7(b). The experimental data clearly demonstrated that the parallel energy of exhausting plasma can be controlled by input RF power only. This feature corresponds to the variable $I_{\text {sp }}$ control demanded in the VASIMR-type thruster. Here $I_{\mathrm{sp}}$ was varied from $1600 \mathrm{sec}$ to $6000 \mathrm{sec}$ in the experiments.

Energy conversion from $W_{\perp}$ to $W_{/ /}$in a diverging magnetic nozzle was confirmed in the hydrogen plasma as well as in the helium plasma. Although the RF input power is low of $6.9 \mathrm{~kW}, T_{i / /}$ attains to more than $50 \mathrm{eV}$ after the nozzle, which corresponds to the specific impulse of $10^{4} \mathrm{sec}$.

\section{Conclusions}

In order to establish a VASIMR-type plasma thruster, ion heating and acceleration experiments were performed in fast-flowing hydrogen and helium plasmas produced by an MPDA in the HITOP device. Strong ion heating occurred by RF wave excitation and energy conversion from perpendicular to parallel direction in a diverging magnetic nozzle was clearly observed in a hydrogen plasma as well as in helium plasma. The ion temperature $T_{\mathrm{i} \perp}$ increased from $2 \mathrm{eV}$ to nearly $60 \mathrm{eV}$ in a hydrogen plasma with the electron density of $1.5 \times 10^{17} \mathrm{~m}^{-3}$. The resonance region of magnetic field was broader than that in a helium plasma. The effect of Doppler shift of ion cyclotron resonance was also observed. The absorption efficiency of the excited wave was higher in a hydrogen plasma than in a helium plasma. In a magnetic nozzle the variation of $T_{\mathrm{i} \perp}$ followed the conservation of the magnetic moment $\mu$, although some discrepancy was observed in larger gradient of magnetic field. It was successfully demonstrated that the parallel energy of exhausting plasma could be changed by controlling the input RF power $P_{\mathrm{RF}}$ without the shaping of magnetic nozzle. The feature of variable $I_{\mathrm{sp}}$ control demanded in the VASIMR-type thruster can be realized not only by shaping the magnetic nozzle configuration but by controlling the RF input power.

\section{Acknowledgments}

This work was supported in part by Grant-in-Aid for Scientific Researches from Japan Society for the Promotion of Science, and under the auspices of the NIFS(National
Institute for Fusion Science) Collaborative Research Program (NIFS07KKMB001).

\section{References}

1) ChangDiaz F.R. et al., : The Physics and Engineering of the VASIMR Engine, Proc. of 36th Joint Propulsion Conference, Huntsville, AIAA-2000-3756, 2000, pp.1-8.

2) Squire J.P., et al.,: High power light gas helicon plasma source for VASIMR, Thin Solid Films, 506 (2006), pp.579-582.

3) Inutake M., et al., "Magnetic-Nozzle Acceleration and Ion Heating of a Supersonic Plasma Flow," Transactions of Fusion Technology, Vol.43, No.1T, FUSTE8, 2002, pp.118-124

4) Ando A., Inutake M., Hatanaka M., Hattori K., Tobari and Yagai T., "Alfven wave excitation and single-pass ion cyclotron heating in a fast-flowing plasma," Physics of Plasmas, Vol.13, 2006, pp.057103 1-7.

5) Ando A., et al., "Ion Heating Experiment by a helical Antenna in a Fast Flowing Plasma," Thin Solid Films, Vol.506, 2006, pp.601-604

6) Ando A., Inutake M., Hattori K., Shibata M., Kasashima Y., "ICRF heating and plasma acceleration with an open magnetic field for the advanced space thruster," Transactions of Fusion Technology, Vol.51, No.2T, FUSTE8, 2007, pp.72-74.

7) Ando A., Shibata M., Kasashima Y., Hattori K. and Inutake M., "Magnetic Nozzle Acceleration in a Fast-flowing Plasma for the VASIMR Thruster," Proceedings of the 25th InternationalSymposium on Space Technology and Science, ISTS 2006-b-42, 2006, pp.292-297.

8) Inutake M., Ando A., Hattori K., Tobari H. Makita T., Shibata M., Y. Kasashima, and Komagome T., "Generation of supersonic plasma flows using an applied-field MPD arcjet and ICRF heating," Plasma Physics and Controlled Fusion, Vol.49, 2007, pp.A121-A134.

9) Inutake M., Ando A., Hattori K., Tobari H. Makita T., Isobe H., and Komagome T., "Transonic plasma flow passing through a magnetic mirror," Transactions of Fusion Technology, Vol.51, No.2T, FUSTE8, 2007, pp.141-146.

10) Tobari H., Ando A., Inutake M., Hattori K., "Characteristics of electromagnetically accelerated plasma flow in an externally-applied magnetic field," Physics of Plasmas, Vol.14, 2007, pp.093507 1-10.

11) Ando A., Kasashima Y., Hagiwara T., Hattori K. and Inutake M., "Ion heating and acceleration experiment in hydrogen plasma for the VASIMR-type Thruster," Proceedings of the 30th International Electric Propulsion Conference, IEPC2007-191, 2007, pp.1-6.

12) Hagiwara T., Ando A., Kasashima Y., Hattori K. and Inutake M., "Experiments of Ion Acceleration in a Magnetic Nozzle for an Advanced Plasma Thruster," Proceedings of the 30th International Electric Propulsion Conference, IEPC-2007255, 2007, pp.1-6. 